\title{
On the tunnel number and the Morse-Novikov number of knots
}

\author{
ANDREI PAJITNOV
}

\begin{abstract}
Let $L$ be a link in $S^{3}$; denote by $\mathcal{M N}(L)$ the Morse-Novikov number of $L$ and by $t(L)$ the tunnel number of $L$. We prove that $\mathcal{M N}(L) \leqslant 2 t(L)$ and deduce several corollaries.
\end{abstract}

57M25, 57M27, 57R35, 57R70; 57R19, 57R45

\section{Introduction}

\subsection{Background}

Let $L$ be a link in $S^{3}$, that is, an embedding of several copies of $S^{1}$ to $S^{3}$. First off, we recall the definition of three numerical invariants of $L$. In the sequel $N(L)$ denotes a closed tubular neighbourhood of $L$.

(A) (Tunnel number) An arc $\gamma$ in $S^{3}$ is called a tunnel for $L$ if $\gamma \cap L$ consists of the two endpoints of $\gamma$. The tunnel number $t(L)$ is the minimal number $m$ of disjoint tunnels $\gamma_{1}, \ldots, \gamma_{m}$ such that the closure of $S^{3} \backslash N\left(L \cup \gamma_{1} \cup \cdots \cup \gamma_{m}\right)$ is a handlebody. The tunnel number was introduced by B Clark [1]; this invariant was studied in the works of T Kohno [11], T Kobayashi [9], T Kobayashi and Y Rieck [10], M Lustig and Y Moriah [13], K Morimoto [15; 14; 16], K Morimoto, M Sakuma and Y Yokota [17; 18], M Scharlemann and J Schultens [23; 24] and others. M Scharlemann and J Schultens [23] proved that $t(n K) \geqslant n$ for any $n$ (here $n K$ stands for the connected sum of $n$ copies of the knot $K)$. They proved also that $t(n K) \geqslant \frac{2}{5} n t(K)$ if $K$ is not a 2-bridge knot [24]. T Kohno [11] gave an estimate of tunnel number of knots in terms of quantum invariants. K Morimoto, M Sakuma and Y Yokota [18] computed the tunnel number of all prime knots with $\leqslant 10$ crossings.

For any two knots $K_{1}, K_{2}$ we have $t\left(K_{1} \# K_{2}\right) \leqslant t\left(K_{1}\right)+t\left(K_{2}\right)+1$. K Morimoto [15] constructed knots $K_{1}, K_{2}$ such that $t\left(K_{1} \# K_{2}\right)<t\left(K_{1}\right)+t\left(K_{2}\right)$. T Kobayashi and Y Rieck [10] define the growth rate for a knot $K$ by the formula

$$
g r_{t}(K)=\limsup _{m \rightarrow \infty} \frac{t(m K)-m t(K)}{m-1} .
$$


It follows from results of [24] that $g r_{t}(K) \geqslant-1-\frac{2}{3} t(K)$.

(B) (Bridge numbers) Let $S^{3}=H_{1} \cup H_{2}$ be a Heegaard splitting of $S^{3}$; put $\Sigma=H_{1} \cap H_{2}$, and $g=g(\Sigma)$. We say (following H Doll [2]) that $L$ is in an $n$-bridge position with respect to $\Sigma$ if $\Sigma$ intersects $L$ in $2 n$ points and $\Sigma \cap H_{i}$ is a union of $n$ trivial arcs in $H_{i}$ for $i=1,2$. The $g$-bridge number $b_{g}(L)$ of $L$ is defined as the minimal number $n$ such that $L$ can be put in an $n$-bridge position with respect to a Heegaard decomposition of genus $g$. Thus $b_{0}(L)$ is the classical bridge number as defined by $\mathrm{H}$ Schubert [25]. We have

$$
t(L) \leqslant g+b_{g}(L)-1 .
$$

(C) (Morse-Novikov numbers) Pick an orientation preserving trivialisation of the normal bundle of $L$. The corresponding diffeomorphism of disc bundles $\phi: L \times D^{2} \rightarrow$ $N(L)$ will be called framing of $L$. Let $C_{L}$ denote the closure of $S^{3} \backslash N(L)$. A Morse function $f: C_{L} \rightarrow S^{1}$ is called regular if its restriction to the boundary $\partial N(L)$ is the canonical fibration over the circle: $(f \circ \phi)(l, z)=z /|z|$. The number of the critical points of index $i$ of a regular Morse function $f$ will be denoted by $m_{i}(f)$; the total number of critical points of $f$ will be denoted by $m(f)$. The minimal value of $m(f)$ over all possible framings $\phi$ and Morse maps $f: C_{L} \rightarrow S^{1}$ is called the Morse-Novikov number of the link $L$ and denoted by $\mathcal{M N}(L)$ (see Veber, Pajitnov and Rudolph [26]).

The Morse-Novikov theory of circle-valued maps (see Novikov [19] and Pajitnov [20; 21]) allows one to obtain homological lower bounds for $\mathcal{M N}(L)$ as follows. Let $\bar{C}_{L}$ be the infinite cyclic covering induced by $f$ from the covering $\mathbb{R} \rightarrow S^{1}$. Denote the ring $\mathbb{Z}\left[t, t^{-1}\right]$ by $\Lambda$, and the ring $\mathbb{Z}((t))$ by $\hat{\Lambda}$. The $\hat{\Lambda}$-module

$$
\mathcal{N}_{*}(L)=H_{*}\left(\bar{C}_{L}\right) \otimes_{\Lambda} \widehat{\Lambda}
$$

is called the Novikov homology of the link $L$. The rank and torsion numbers of the $\widehat{\Lambda}$-module $\mathcal{N}_{1}(L)$ are denoted respectively by $b_{1}(L)$ and $q_{1}(L)$. We have then [26]

$$
\mathcal{M N}(L) \geqslant 2\left(b_{1}(L)+q_{1}(L)\right)
$$

In case when the Novikov numbers are not sufficient to determine the $\mathcal{M N}(L)$ the twisted Novikov numbers (introduced by H Goda and the author in [5]) are useful.

As for upper bounds for $\mathcal{M N}(L)$, not much is known. H Goda announced in [4] that $\mathcal{M N}(L) \leqslant 2$ for every prime link $L$ with $\leqslant 10$ crossings. M Hirasawa proved that for every 2-bridge knot $K$ we have $\mathcal{M N}(K) \leqslant 2$ (unpublished). In the papers [22; 7] of L Rudolph and M Hirasawa it is proved that $\mathcal{M N}(K) \leqslant 4 g_{f}(K)$ where $g_{f}(K)$ is the free genus of $K$, that is, the minimal possible genus of a Seifert surface $\Sigma$ bounding $K$ such that $S^{3} \backslash \Sigma$ is an open handlebody. 


\subsection{Main results}

The main result of this work is the following theorem.

Theorem 1.1 For every link $L$ in $S^{3}$ we have

$$
\mathcal{M N}(L) \leqslant 2 t(L) \text {. }
$$

The following corollaries are easily deduced.

Corollary 1.2 For every $g$ we have

$$
\mathcal{M N}(L) \leqslant 2\left(g+b_{g}(L)-1\right) .
$$

Corollary 1.3 For every tunnel number 1 knot $K$ we have $\mathcal{M N}(K) \leqslant 2$. In particular this holds for any $(1,1)-k n o t K$.

Corollary 1.4 For every link $L$ we have

$$
q_{1}(L)+b_{1}(L) \leqslant t(L) .
$$

Corollary 1.5 For every knot $K$

$$
g r_{t}(K) \geqslant-t(K)+q_{1}(K) .
$$

\section{Proof of Theorem 1.1}

Let $m=t(L)$. Pick a framing $\phi: L \times D^{2} \rightarrow N(L)$. Then the manifold $C_{L}=$ $\overline{S^{3} \backslash N(L)}$ is obtained from $\partial C_{L}$ by attaching $m$ one-handles and then attaching a handlebody of genus $m+1$ to the resulting cobordism. So we obtain a Morse function $g: C_{L} \rightarrow \mathbb{R}$ which is constant on $\partial C_{L}$ and has the following Morse numbers: $m_{0}(g)=$ $0, m_{1}(g)=m, m_{2}(g)=m+1, m_{3}(g)=1$. Pick any Morse map $h: C_{L} \rightarrow S^{1}$ such that $h \mid \partial C_{L}$ is the canonical fibration: $(h \circ \phi)(l, z)=z /|z|$. The 1 -form induced by $h$ from the canonical volume form on $S^{1}$ will be denoted by $d h$. Consider a closed 1 -form $\omega_{\epsilon}=d g+\epsilon d h$. For $\epsilon>0$ sufficiently small $\omega_{\epsilon}$ is a Morse form with the same Morse numbers as $d g$. The De Rham cohomology class of the 1-form

$$
\frac{1}{\epsilon} \omega_{\epsilon}=\frac{1}{\epsilon} d g+d h
$$

is the same as that of $d h$; therefore this form is the differential of a Morse map $g_{1}: C_{L} \rightarrow S^{1}$ homotopic to $h .{ }^{1}$ Observe that the map $g_{1}$ is a regular Morse map;

\footnotetext{
${ }^{1}$ A similar perturbation argument was used by J C Sikorav in another context; see Pajitnov [20].
} 
it has one local maximum, and the standard elimination procedure (see for example Lemmas 3.1 and 3.2 of [26] for details) gives us a regular Morse function $f: C_{L} \rightarrow S^{1}$ with $m_{0}(f)=0, m_{1}(f) \leqslant m, m_{2}(f) \leqslant m, m_{3}(f)=0$. Thus $\mathcal{M N}(L) \leqslant 2 m$.

\section{Examples}

Theorem 1.1 can be used in two ways. A lot of information is available about the tunnel numbers, and this implies new estimates for the Morse-Novikov numbers of knots. On the other hand, the Novikov torsion number $q_{1}(K)$ is an invariant which is easy to compute, and in many cases this gives new information about the sequence of tunnel numbers $t(n K)$ for a given knot $K$. Let us consider two examples:

(A) (Pretzel knots) Let $q, r$ be positive integers; denote by $\mathcal{P}$ the $(2 r+1)$-stranded pretzel knot $P(2 q+1,-2 q-1,2 q+1, \ldots, 2 q+1)$. The knot $\mathcal{P}$ for $q=1, r=2$ is depicted below.

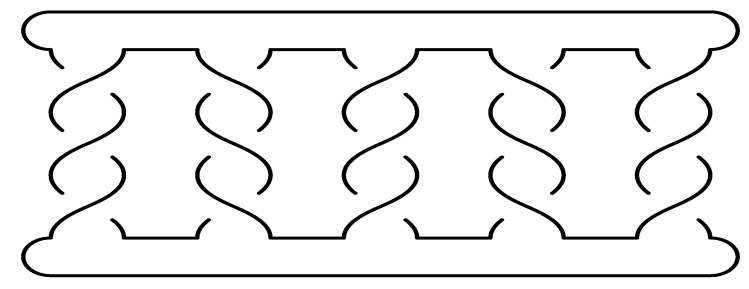

Figure 1: Pretzel knot

It is clear that $t(\mathcal{P}) \leqslant 2 r$. An easy computation of the Alexander module via the Seifert matrix gives

$$
\mathcal{N}_{1}(\mathcal{P}) \approx(\widehat{\Lambda} / X Y \hat{\Lambda})^{r}
$$

where $X=q t-(q+1), Y=(q+1) t-q$. Thus $q_{1}(\mathcal{P})=r$. Since $q_{1}(m K)=m q_{1}(K)$ for any knot $K$, we deduce that

$$
\frac{1}{2} n t(\mathcal{P}) \leqslant n q_{1}(\mathcal{P}) \leqslant t(n \mathcal{P})
$$

In particular the growth rate of the knot satisfies $g r_{t}(K) \geqslant-\frac{1}{2} t(K)$.

(B) (A twisted $\left.5_{2} \# 5_{2}\right)$ Let $K$ be the knot obtained from the connected sum $5_{2} \# 5_{2}$ by twisting (see Figure 2).

An easy computation shows that

$$
\mathcal{N}_{1}(K) \approx(\widehat{\Lambda} / S \widehat{\Lambda})^{2}
$$




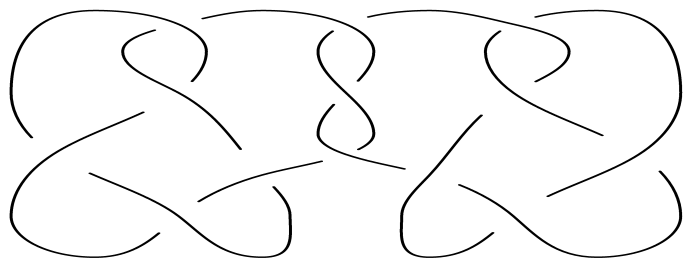

Figure 2: The twisted $5_{2} \# 5_{2}$ knot

where $S=2 t^{2}-3 t+2$ is the Alexander polynomial of the knot $5_{2}$. Thus $q_{1}(K)=2$. Since $t(K) \leqslant 3$ we obtain

$$
\frac{2}{3} n t(K) \leqslant n q_{1}(K) \leqslant t(n K) .
$$

We have therefore $g r_{t}(K) \geqslant-\frac{1}{3} t(K)$.

\section{Relations with previously known results}

A theorem of M Hirasawa says that $\mathcal{M N}(K) \leqslant 2$ if $K$ is a two-bridge knot. Since $t(K) \leqslant b(K)-1$ our theorem implies this result. Observe that M Hirasawa's proof uses H Schubert's presentation of 2-bridge knots, and can not be generalized to the case of arbitrary bridge number.

The inequality (1) implies also the upper bound

$$
\mathcal{M N}(K) \leqslant 4 g_{f}(K)
$$

obtained by L Rudolph and M Hirasawa [22; 7]. Indeed, J H Lee [12] has shown that $t(K) \leqslant 2 g_{f}(K)$.

In many cases the estimate of Theorem 1.1 is better than the free genus estimate. For example, let $K$ be the pretzel knot $K=P(-2 l, q, r)$ where $l \geqslant 2$ and $q, r \geqslant 3$ are odd numbers. Then $t(K) \leqslant 2$, and the Alexander polynomial of the knot equals

$$
A(t)=l t^{q+r}-(2 l-1) t^{q+r-1}+\cdots-(2 l-1) t+l
$$

(see the work [8] of D Kim and J Lee). Therefore $K$ is not fibred, and $4 \geqslant \mathcal{M N}(K) \geqslant 2$. As for the genus of $K$, we have $g(K) \geqslant \operatorname{deg} A(t) / 2=(q+r) / 2$, therefore the free genus of $K$ is not less than $(q+r) / 2$. 
Theorem 1.1 leads to quick proofs of results about the Morse-Novikov numbers already known. The simplest cases are: the link $A_{n}$ (the boundary of $n$-twisted unknotted annulus) and the twist knots $K_{n}$. See Figures 3 and 4 . We shall assume that $n \geqslant 2$.

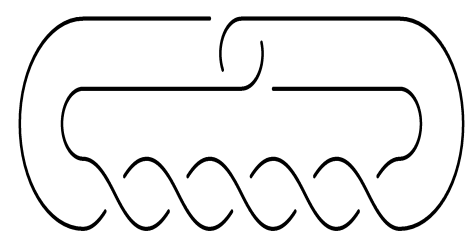

Figure 3: The knot $K_{2}$

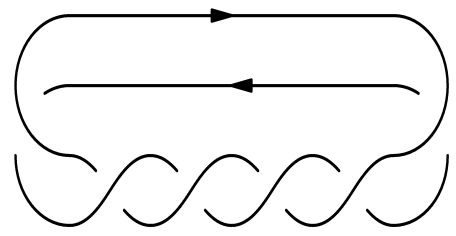

Figure 4: The link $A_{2}$

Since the tunnel number of these links equals 1 we have $\mathcal{M N}\left(A_{n}\right) \leqslant 2, \mathcal{M N}\left(K_{n}\right) \leqslant 2$. It is easy to show that $q_{1}\left(K_{n}\right)=q_{1}\left(A_{n}\right)=1[26 ; 6]$, thus

$$
\mathcal{M N}\left(A_{n}\right)=2, \mathcal{M N}\left(K_{n}\right)=2 .
$$

In the paper [4] H Goda announced the computation of the Morse-Novikov numbers of all prime knots and links with $\leqslant 10$ crossings. His theorem (which is based on the results of [3]) says that for every nonfibred prime link $L$ with $\leqslant 10$ crossings we have $\mathcal{M N}(L)=2$.

Since the tunnel numbers of prime knots with $\leqslant 10$ crossings are known from the work of K Morimoto, M Sakuma and S Yokota [18], our Theorem 1.1 provides a quick proof of H Goda's results at least for knots with $\leqslant 8$ crossings. Indeed, it is proved in [18] that among the prime knots with $\leqslant 8$ crossings only the knots $8_{16}, 8_{17}, 8_{18}$ have the tunnel number 2 ; the tunnel number of all the others equals 1 . Since these three knots are fibred, we deduce that every nonfibred prime knot with $\leqslant 8$ crossings has the tunnel number equal to 1 and therefore its Morse-Novikov number is equal to 2 . 


\section{Open questions and further remarks}

(1) One of the main conjectures in the Morse-Novikov theory of knots and links is the following (M Boileau, C Weber):

$$
\mathcal{M N}\left(K_{1} \# K_{2}\right)=\mathcal{M N}\left(K_{1}\right)+\mathcal{M N}\left(K_{2}\right) .
$$

The example of K Morimoto [15] shows that there are knots $K_{1}, K_{2}$ with $t\left(K_{1} \# K_{2}\right)<$ $t\left(K_{1}\right)+t\left(K_{2}\right)$. Moreover, T Kobayashi [9] proved that for every $N$ there are knots $K_{1}$ and $K_{2}$ such that $t\left(K_{1} \# K_{2}\right) \leqslant t\left(K_{1}\right)+t\left(K_{2}\right)-N$. In view of the relations between the tunnel and the Morse-Novikov numbers established in the present paper, these results provide a number of potential counterexamples to the conjecture (2).

(2) The Novikov homology $\mathcal{N}_{*}(K)$ can be considered as homology with local coefficients with respect to the representation

$$
\mu: \pi_{1}\left(C_{K}\right) \rightarrow \mathbb{Z}[\mathbb{Z}]^{\times}=\Lambda^{\times} \subset \hat{\Lambda}^{\times}=\operatorname{GL}(1, \hat{\Lambda}),
$$

where the first arrow is the meridian homomorphism $\pi_{1}\left(C_{K}\right) \rightarrow \mathbb{Z} \subset \mathbb{Z}[\mathbb{Z}]^{\times}$. Thus Corollary 1.4 can be reformulated as follows:

$$
t(K) \geqslant \mathrm{m}_{\widehat{\Lambda}}\left(H_{1}\left(C_{K}, \mu\right)\right)
$$

where $\mathrm{m}_{\hat{\Lambda}}(N)$ stands for the minimal number of generators over $\widehat{\Lambda}$ of the module $N$. For an arbitrary representation we have a weaker (obvious) inequality:

Proposition 5.1 For every representation $\rho: \pi_{1}\left(C_{K}\right) \rightarrow \operatorname{GL}(n, R)$ (where $R$ is a principal ring) we have

$$
t(K) \geqslant \frac{1}{n}\left(\mathrm{~m}_{R}\left(H_{1}\left(C_{K}, \rho\right)\right)\right)-1 .
$$

Question Is it true that

$$
t(K)=\max _{\rho}\left(\frac{1}{n}\left(\mathrm{~m}_{R}\left(H_{1}\left(C_{K}, \rho\right)\right)\right)-1\right) ?
$$

In other words, is the information deduced from the twisted homology sufficient to determine the tunnel number of any knot?

Acknowledgements This work was completed during my stay at the Graduate Mathematical School of the Tokyo University. I am grateful to T Kohno for a warm hospitality and many stimulating discussions during my stay.

Many thanks to T Kobayashi and Y Rieck for detailed discussions on the tunnel numbers and related topics. 


\section{References}

[1] B Clark, The Heegaard genus of manifolds obtained by surgery on links and knots, Internat. J. Math. Math. Sci. 3 (1980) 583-589 MR582900

[2] H Doll, A generalized bridge number for links in 3-manifolds, Math. Ann. 294 (1992) 701-717 MR1190452

[3] H Goda, On handle number of Seifert surfaces in $S^{3}$, Osaka J. Math. 30 (1993) 63-80 MR1200821

[4] H Goda, Some estimates of the Morse-Novikov numbers for knots and links, from: "Intelligence of low dimensional topology 2006", (J S Carter, S Kamada, L H Kauffman, A Kawauchi, T Kohno, editors), Ser. Knots Everything 40, World Sci. Publ., Hackensack, NJ (2007) 35-42 MR2371706

[5] H Goda, A V Pajitnov, Twisted Novikov homology and circle-valued Morse theory for knots and links, Osaka J. Math. 42 (2005) 557-572 MR2166722

[6] H Goda, A V Pajitnov, Dynamics of gradient flows in the half-transversal Morse theory, Proc. Japan Acad. Ser. A Math. Sci. 85 (2009) 6-10 MR2488751

[7] M Hirasawa, L Rudolph, Constructions of Morse maps for knots and links, and upper bounds on the Morse-Novikov number, to appear in J. Knot Theory Ramifications arXiv:math.GT/0311134

[8] D Kim, J Lee, Some invariants of pretzel links, Bull. Austral. Math. Soc. 75 (2007) 253-271 MR2312569

[9] T Kobayashi, A construction of arbitrarily high degeneration of tunnel numbers of knots under connected sum, J. Knot Theory Ramifications 3 (1994) 179-186 MR1279920

[10] T Kobayashi, Y Rieck, On the growth rate of the tunnel number of knots, J. Reine Angew. Math. 592 (2006) 63-78 MR2222730

[11] T Kohno, Tunnel numbers of knots and Jones-Witten invariants, from: "Braid group, knot theory and statistical mechanics, II", (C N Yang, M L Ge, editors), Adv. Ser. Math. Phys. 17, World Sci. Publ., River Edge, NJ (1994) 275-293 MR1338606

[12] J H Lee, An upper bound for tunnel number of a knot using free genus, Lecture notes, 4-th East Asian School of knots (2008) Available at http:// faculty.ms.u-tokyo.ac.jp/ topology/EAS4slides/JungHoonLee.pdf

[13] M Lustig, Y Moriah, Generalized Montesinos knots, tunnels and $\mathcal{N}$-torsion, Math. Ann. 295 (1993) 167-189 MR1198847

[14] K Morimoto, On the additivity of tunnel number of knots, Topology Appl. 53 (1993) 37-66 MR1243869

[15] K Morimoto, There are knots whose tunnel numbers go down under connected sum, Proc. Amer. Math. Soc. 123 (1995) 3527-3532 MR1317043 
[16] K Morimoto, On the super additivity of tunnel number of knots, Math. Ann. 317 (2000) 489-508 MR1776114

[17] K Morimoto, M Sakuma, Y Yokota, Examples of tunnel number one knots which have the property " $1+1=3$ ”, Math. Proc. Cambridge Philos. Soc. 119 (1996) 113-118 MR1356163

[18] K Morimoto, M Sakuma, Y Yokota, Identifying tunnel number one knots, J. Math. Soc. Japan 48 (1996) 667-688 MR1404816

[19] S P Novikov, Multivalued functions and functionals. An analogue of the Morse theory, Dokl. Akad. Nauk SSSR 260 (1981) 31-35 MR630459

[20] A V Pajitnov, On the Novikov complex for rational Morse forms, Ann. Fac. Sci. Toulouse Math. (6) 4 (1995) 297-338 MR1344724

[21] A V Pajitnov, Circle-valued Morse theory, de Gruyter Studies in Math. 32, de Gruyter, Berlin (2006) MR2319639

[22] L Rudolf, Murasugi sums of Morse maps to the circle, Morse-Novikov numbers, and free genus of knots arXiv:math.GT/0108006

[23] M Scharlemann, J Schultens, The tunnel number of the sum of $n$ knots is at least $n$, Topology 38 (1999) 265-270 MR1660345

[24] M Scharlemann, J Schultens, Annuli in generalized Heegaard splittings and degeneration of tunnel number, Math. Ann. 317 (2000) 783-820 MR1777119

[25] H Schubert, Über eine numerische Knoteninvariante, Math. Z. 61 (1954) 245-288 MR0072483

[26] K Veber, A V Pajitnov, L Rudolf, The Morse-Novikov number for knots and links, Algebra i Analiz 13 (2001) 105-118 MR1850189

Laboratoire Mathématiques Jean Leray UMR 6629, Université de Nantes Faculté des Sciences, 2, rue de la Houssinière, 44072 Nantes Cedex, France andrei.pajitnov@gmail.com, Andrei.Pajitnov@univ-nantes.fr http://www.math.sciences.univ-nantes.fr/ pajitnov/

Received: 19 October 2009 